\title{
A meshless solution of two dimensional density-driven groundwater flow
}

\author{
K. Kovarik \\ Faculty of Civil Engineering, University of Zilina, Slovakia
}

\begin{abstract}
The boundary element method (BEM) is a very useful method used for numerical models of groundwater flow. However, this method was aimed to solve problems in homogeneous domains and it presents even greater difficulties than the other numerical methods when coping with the nonhomogeneities which are so characteristic in the groundwater hydraulics. The meshless LBIE method is a very promising meshless scheme. This method is characterized as meshless since distributed nodal points, covering the domain, are employed. Nodal points can be randomly spread over the domain. Every node is surrounded by a simple surface (circle) centered at the collocation point and the boundary integral equation is written on this local boundary. The unknown variables, in the local sub-domains, are approximated by some of the interpolation methods to obtain a system of linear equations. Solving this system of equations leads to the numerical solution for the main problem. Several authors used the moving least squares (MLS) method as an interpolation method but nowadays the radial basis functions (RBF) interpolation is used instead. In this paper the combination of RBF and the dual reciprocity method is used to solve the time-dependent groundwater flow in heterogeneous domain combined with temperature transport which also influences the density and viscosity of groundwater.
\end{abstract}

Keywords: heat transfer, density driven flow, dual reciprocity, radial basis functions.

\section{Introduction}

The density driven groundwater flow occurs mainly in some environmental problems such as saltwater intrusion or leakage from landfills. We must solve this problem also when we study the geothermal problems. Although density driven 
flow problems are mainly three-dimensional, they are often simulated by vertical 2D numerical models due to their very high computational costs.

The boundary element method and dual reciprocity method (DRM) are very useful methods used for numerical models of groundwater flow (see e.g. [1]). However, these method was mainly aimed to solve problems in homogeneous domains and it presents some difficulties when coping with the non homogeneities which are so characteristic of the groundwater hydraulics. The disadvantage can be overcome using some meshless scheme combined with BEM or DRM. The meshless LBIE method, introduced by Zhu et al. [2], is a very promising meshless scheme. This method is characterized as meshless since distributed nodal points, covering the domain, are employed. Nodal points are randomly spread over the domain. Every node is surrounded by a simple surface (circle) centered at the collocation point and the boundary integral equation is written on this local boundary. The unknown variables, in the local sub-domains, are approximated by some of the interpolation method to obtain a system of linear equations. Solving this system of equations leads to the numerical solution for the main problem. Zhu et al. [2] used the moving least squares (MLS) method as an interpolation method but nowadays the RBF interpolation is used instead (see e.g. [3]). In this paper the solution of coupled groundwater flow-heat transfer problem based on this RBIEM method is presented.

\section{Governing equations}

A density-driven groundwater flow can be described by the following equation (see also [4])

$$
\rho S \frac{\partial h}{\partial t}+\epsilon \frac{\partial \rho}{\partial t}+\nabla(\rho \vec{q})=0
$$

where $\mathrm{h}$ is the groundwater potential, $\epsilon$ is the porosity of porous medium, $\rho$ is the density of solution and $\vec{q}$ is the flux defined by

$$
\mathbf{q}=-\mathbf{K}\left(\nabla h+\frac{\rho-\rho_{0}}{\rho_{0}} \nabla \vec{x}\right)
$$

where $\mathbf{K}$ is matrix of hydraulic conductivities and $\rho_{0}$ initial density of water. The differential equation of $2 \mathrm{D}$ groundwater flow with variable density is now expressed as

$$
\frac{\partial}{\partial x}\left(\rho K_{x} \frac{\partial h}{\partial x}\right)+\frac{\partial}{\partial y}\left[\rho K_{y}\left(\frac{\partial h}{\partial y}+\frac{\rho-\rho_{0}}{\rho_{0}}\right)\right]=\epsilon \frac{\partial \rho}{\partial t}+\rho S \frac{\partial h}{\partial t}
$$

where we denote $K_{x}, K_{y}$ hydraulic conductivities $\left[L T^{-1}\right]$ in direction $x$ and $y$, respectively. Heat transfer in porous media can be described by the following 
equation (see e.g. [5])

$$
\frac{\partial}{\partial x_{i}}\left(K_{T B} \frac{\partial T}{\partial x_{j}}-q_{i} \rho_{L} c_{L} T\right)=\rho_{B} c_{B} \frac{\partial T}{\partial t}
$$

where $T$ is temperature, $K_{T}$ is thermal conductivity, $c$ is specific heat capacity. The subscripts $L$ and $B$ refer to liquid and bulk phases, respectively.

\section{Dual reciprocity formulation}

The Eq. (3) can be transformed to the following shape

$$
\begin{array}{r}
\frac{\partial^{2} h}{\partial x^{2}}+\frac{K_{y}}{K_{x}} \frac{\partial^{2} h}{\partial y^{2}}=\frac{\epsilon}{K_{x} \rho} \frac{\partial \rho}{\partial t}+\frac{S}{K_{x}} \frac{\partial h}{\partial t}-\frac{1}{\rho} \frac{\partial \rho}{\partial x} \frac{\partial h}{\partial x}- \\
-\frac{K_{y}}{K_{x} \rho} \frac{\partial \rho}{\partial y} \frac{\partial h}{\partial y}-\frac{K_{y}}{K_{x} \rho \rho_{0}} \frac{\partial}{\partial y}\left(\rho^{2}-\rho \rho_{0}\right)
\end{array}
$$

To solve Eq. (5) in the domain $\Omega$ with boundary $\Gamma$ using the DRM we should use the following transformation of co-ordinates

$$
\tilde{x}=x \quad \tilde{y}=y \sqrt{\frac{K_{x}}{K_{y}}}
$$

and we get

$$
\begin{gathered}
\frac{\partial^{2} h}{\partial \tilde{x}^{2}}+\frac{\partial^{2} h}{\partial \tilde{y}^{2}}=\frac{\epsilon}{K_{x} \rho} \frac{\partial \rho}{\partial t}+\frac{S}{K_{x}} \frac{\partial h}{\partial t}-\frac{1}{\rho} \frac{\partial \rho}{\partial \tilde{x}} \frac{\partial h}{\partial \tilde{x}}- \\
-\frac{1}{\rho} \sqrt{\frac{K_{y}}{K_{x}}} \frac{\partial \rho}{\partial \tilde{y}} \frac{\partial h}{\partial \tilde{y}}-\frac{1}{\rho \rho_{0}} \sqrt{\frac{K_{y}}{K_{x}}} \frac{\partial}{\partial \tilde{y}}\left(\rho^{2}-\rho \rho_{0}\right)
\end{gathered}
$$

Applying the Green integral formula to the left side of Eq. (7) we get the following integral form

$$
c_{k} h_{k}+\int_{\Gamma} \frac{\partial u_{k j}^{*}}{\partial n} h_{j} d \Gamma-\int_{\Gamma} u_{k j}^{*} \frac{\partial h_{j}}{\partial n} d \Gamma-\int_{\Omega} u_{k j}^{*} b_{j} d \Omega=0
$$

Here $b_{j}$ is the value of the right side of Eq. (7) in point $\mathrm{j}$ and $u_{k j}^{*}$ is the fundamental solution of Laplace equation. The constant $c_{k}$ has value from 0 to 1 being 0.5 if the point $\mathrm{k}$ is placed on the smooth boundary. The DRM is used to transform the domain integral in Eq. (8) to equivalent boundary integrals (see [6]). The basic idea is to expand the term $b_{j}$ using the approximation

$$
b_{j} \cong \sum_{i=1}^{N_{b}+N_{i}} \alpha_{i} f_{i j}
$$

where $\alpha_{i}$ is a set of initially unknown coefficients and $f_{i j}$ are approximating functions. The approximation employs $N_{b}$ nodes on the boundary $\Gamma$ and $N_{i}$ points 
inside the domain $\Omega$. The functions $f_{i j}$ in Eq. (9) belongs to the family of radial basis function. The most often used functions are

$$
f_{i j}=1+\sum_{m=1}^{n} r_{i j}^{m}
$$

Now we can define the series of particular solutions $\hat{h}_{i j}$. These solutions and the approximating functions $f_{i j}$ are linked through the equation

$$
\frac{\partial^{2} \hat{h}_{i j}}{\partial \tilde{x}^{2}}+\frac{\partial^{2} \hat{h}_{i j}}{\partial \tilde{y}^{2}}=f_{i j}
$$

Applying the Green's formula to the Eq. (11) and using Eq. (9) we can transform Eq. (8) and get the boundary only integral formula

$$
\begin{array}{r}
c_{k} h_{k}+\int_{\Gamma} \frac{\partial u_{k j}^{*}}{\partial n} h_{j} d \Gamma-\int_{\Gamma} u_{k j}^{*} \frac{\partial h_{j}}{\partial n} d \Gamma= \\
=\sum_{i=1}^{N_{b}+N_{i}} \alpha_{i}\left(c_{k} \hat{h}_{k i}+\int_{\Gamma} \frac{\partial u_{k i}^{*}}{\partial n} \hat{h}_{i j} d \Gamma-\int_{\Gamma} u_{k i}^{*} \frac{\partial \hat{h}_{i j}}{\partial n} d \Gamma\right)
\end{array}
$$

The discretized form of Eq. (12) for source point $\mathrm{k}$ can be written as

$$
\begin{array}{r}
c_{k} h_{k}+\sum_{j=1}^{N_{b}} H_{k j} h_{j}-\sum_{j=1}^{N_{b}} G_{k j} q_{j}= \\
=\sum_{i=1}^{N_{b}+N_{i}} \alpha_{i}\left(c_{k} \hat{h}_{k i}+\sum_{j=1}^{N_{b}} H_{k i} \hat{h}_{i j}-\sum_{j=1}^{N_{b}} G_{k i} \hat{q}_{i j}\right)
\end{array}
$$

where $H_{k j}$ and $G_{k j}$ are matrices obtained from the integrations of $q^{*}$ and $u^{*}$ at each boundary element. The unknown coefficients $\alpha_{i}$ can be determined from Eq. (9) as

$$
\alpha_{i}=\sum_{j=1}^{N_{b}+N_{i}} F_{i j}^{-1} b_{j}
$$

where $F_{i j}^{-1}$ are members of the inverse matrix of values of function $f_{i j}$.

\section{Radial basis functions interpolation}

Radial basis functions (RBF) are initially known as a powerful tool for approximating multivariate functions on a scattered data. Due to their mesh-free nature RBF have received an increasing attention for solving partial differential equations (PDE) of different kinds. The first trial of such exploration was made by Kansa [7]. Full exploitation of the RBF method was constrained by the progressive 
ill-conditioned coefficient matrix as the number of nodes increases. To remove this difficulty, Shu et al. (see [8]) suggested using the local RBF method in which the approximation is formed by using only several local supporting points. The unknown function $U$ is approximated in a sub-domain which forms the neighborhood or support of a reference point $i$ by weighted sum of multiquadric functions and polynomials

$$
U\left(x_{i}, y_{i}\right)=\sum_{j=1}^{n} \lambda_{j} R\left(r_{i j}\right)+\sum_{j=1}^{m} \chi_{j} p_{j}\left(x_{i}, y_{i}\right)
$$

where $\lambda_{j}$ and $\chi_{j}$ are weights, $R\left(r_{i j}\right)$ are the RBF basis functions, and $p_{j}$ is a basis for polynomial space with degree $m-1, m$ is the order of $R$ and $n$ is a number of field nodes in the neighborhood of a reference point. Multiquadric functions are one of the most popular radial functions used for this purpose and they are defined as

$$
R\left(r_{i j}\right)=\sqrt{r_{i j}^{2}+\epsilon^{2}}
$$

where $r_{i j}$ is a distance between points $i$ and $j$ and $\epsilon$ is a so-called shape factor of multiquadric function. Coefficients $\lambda_{j}, \chi_{j}$ in Eq. (15) can be determined by enforcing Eq. (15) to be satisfied at these $N_{i}$ nodes surrounding the point of interest. This leads to $n$ linear equations, one for each node. The matrix form of these equations can be expressed as

$$
\mathbf{U}=\mathbf{R} \lambda+\mathbf{P} \chi
$$

where $\mathbf{U}=\left[U_{1}, U_{2}, \ldots U_{N_{i}}\right]$ is a vector of function values in all supporting nodes. However there are $n+m$ variables in this equation. The additional $m$ equations can be added using the following $m$ constraint conditions

$$
\sum_{i=1}^{n} p_{j}\left(x_{i}, y_{i}\right) \lambda_{i}=0, \quad j=1 \ldots m
$$

Combining Eqs.(17) and (18) yields the following set of equations in the matrix form

$$
\mathbf{u}=\mathbf{A} \mathbf{a}
$$

where we denoted

$$
\mathbf{A}=\left[\begin{array}{cc}
\mathbf{R} & \mathbf{p} \\
\mathbf{p}^{T} & \mathbf{0}
\end{array}\right], \quad \mathbf{a}=\left\{\begin{array}{c}
\lambda \\
\chi
\end{array}\right\}, \quad \mathbf{u}=\left\{\begin{array}{c}
\mathbf{U} \\
\mathbf{0}
\end{array}\right\}
$$

The order $m$ of multiquadric functions is equal to one and, therefore, we need one additional condition to make the interpolation problem well-posed. This 
condition is

$$
\sum_{j=1}^{n} \lambda_{j}=0
$$

Solving Eq. (19) we get

$$
\mathbf{a}=\mathbf{A}^{-1} \mathbf{u}
$$

Eq. (15) can be now written as (see e.g. [9])

$$
U\left(x_{i}, y_{i}\right)=\left[\mathbf{R}^{T}\left(x_{i}, y_{i}\right) \mathbf{p}^{T}\left(x_{i}, y_{i}\right)\right] \mathbf{a}=\left[\mathbf{R}^{T}\left(x_{i}, y_{i}\right) \mathbf{p}^{T}\left(x_{i}, y_{i}\right)\right] \mathbf{A}^{-1} \mathbf{u}
$$

The first $n$ members of the product $\left[\mathbf{R}^{T}\left(x_{i}, y_{i}\right) \mathbf{p}^{T}\left(x_{i}, y_{i}\right)\right] \mathbf{A}^{-1}$ create set of $\mathrm{RBF}$ shape functions $\phi_{j}$ and Eq. (23) can be rewritten as

$$
U\left(x_{i}, y_{i}\right)=\sum_{j=1}^{n} \phi_{j} U_{j}
$$

This formula is used to develop the meshless local DRM in the next section.

\section{Meshless local DRM formulation}

In this section we follow the principle of RBIEM (see [3]). The area of interest $\Omega$ is covered by single nodes on the global boundary $\Gamma$ and also inside the area (see Fig. 1). The local network of 16 elements was created around every node. This simplifies the generation of local boundaries and evaluation of integrals. Next four virtual internal points was added to every network (see Fig. 2) because the solution is usually more accurate (see [6]). The values of potential and its derivatives in

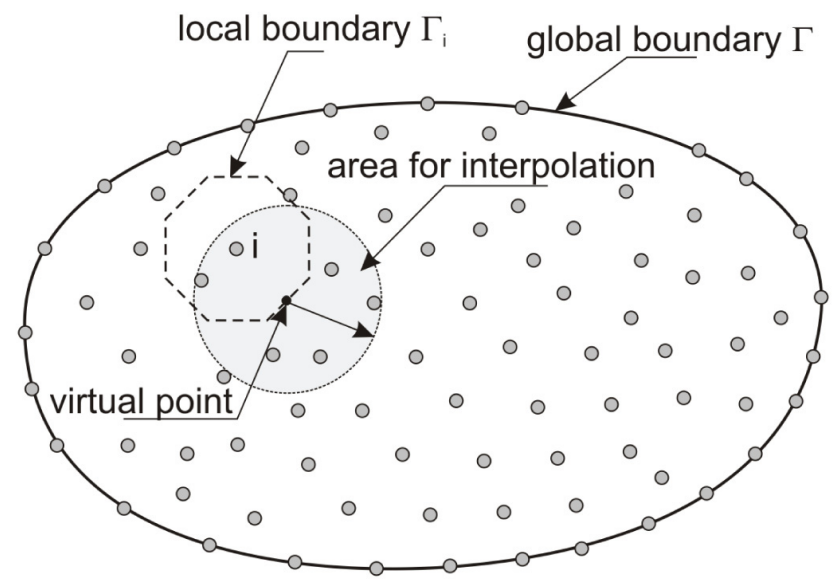

Figure 1: Nodes in the global area. 


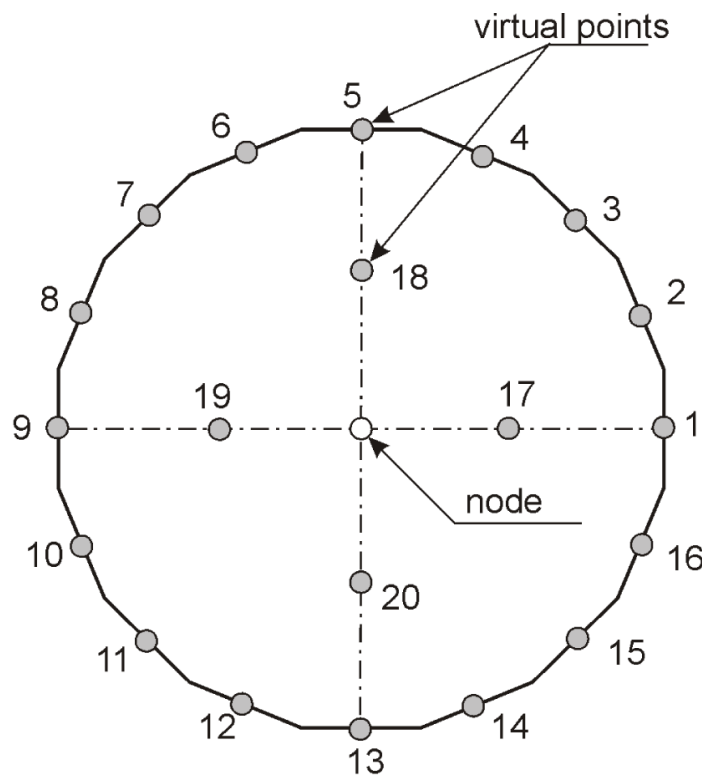

Figure 2: Local network.

$x$ and $y$ directions in node $k$ can be computed from values of potentials and derivatives in virtual points using Eq. (9) as

$$
\begin{gathered}
h_{k}=\sum_{j=1}^{N_{v b}} G_{i j} q_{x j} n_{x}+\sum_{j=1}^{N_{v b}} G_{i j} q_{y j} n_{y}-\sum_{j=1}^{N_{v b}} H_{i j} h_{j}+ \\
+\sum_{k=1}^{N_{v b}+N_{v i}}\left\{\alpha_{k}\left(c_{i} \hat{h}_{i k}+\sum_{j=1}^{N_{v b}} H_{i k} \hat{h}_{k j}-\sum_{j=1}^{N_{v b}} G_{i k} \hat{q}_{k j}\right)\right\} \\
\frac{\partial h_{k}}{\partial x}=\sum_{j=1}^{N_{v b}} \frac{\partial G_{i j}}{\partial x} q_{x j} n_{x}+\sum_{j=1}^{N_{v b}} \frac{\partial G_{i j}}{\partial x} q_{y j} n_{y}-\sum_{j=1}^{N_{v b}} \frac{\partial H_{i j}}{\partial x} h_{j}+ \\
+\sum_{k=1}^{N_{v b}+N_{v i}}\left\{\alpha_{k}\left(\frac{\partial \hat{h}_{i k}}{\partial x}+\sum_{j=1}^{N_{v b}} \frac{\partial H_{i k}}{\partial x} \hat{h}_{k j}-\sum_{j=1}^{N_{v b}} \frac{\partial G_{i k}}{\partial x} \hat{q}_{k j}\right)\right\}
\end{gathered}
$$

where $N_{v b}$ is the number of virtual boundary points, $N_{v i}$ is the number of virtual internal points, $h_{j}$ are the values of potential in virtual points, $q_{x j}, q_{y j}$ are the derivatives in boundary virtual points in $x$ and $y$ directions and $n_{x}, n_{y}$ are the directional cosines of outer normal to virtual boundary, respectively. The derivative in direction y is computed according similar formula to Eq. (26). Values 
of potential and its derivatives can be approximated using RBF interpolation according Eq. (24) and we get

$$
\begin{gathered}
h_{k}=\sum_{j=1}^{N_{v b}} G_{i j} n_{x} \sum_{m=1}^{n} \phi_{m} q_{x m}+\sum_{j=1}^{N_{v b}} G_{i j} n_{y} \sum_{m=1}^{n} \phi_{m} q_{y m}- \\
-\sum_{j=1}^{N_{v b}} H_{i j} \sum_{m=1}^{n} \phi_{m} h_{m}+ \\
+\sum_{k=1}^{N_{v b}+N_{v i}}\left\{\alpha_{k}\left(c_{i} \hat{\Phi}_{i k}+\sum_{j=1}^{N_{v b}} H_{i k} \hat{\Phi}_{k j}-\sum_{j=1}^{N_{v b}} G_{i k} \hat{q}_{k j}\right)\right\} \\
\frac{\partial h_{k}}{\partial x}=\sum_{j=1}^{N_{v b}} \frac{\partial G_{i j}}{\partial x} n_{x} \sum_{m=1}^{n} \phi_{m} q_{x m}+\sum_{j=1}^{N_{v b}} \frac{\partial G_{i j}}{\partial x} n_{y} \sum_{m=1}^{n} \phi_{m} q_{y m}- \\
+\sum_{k=1}^{N_{v b}+N_{v i}}\left\{\begin{array}{c}
\left.\alpha_{k}\left(\frac{\partial \hat{h}_{i k}}{\partial x}+\sum_{j=1}^{N_{v b}} \frac{\partial H_{i k}}{\partial x} \hat{h}_{k j}-\sum_{j=1}^{N_{v b}} \frac{\partial H_{i k}}{\partial x} \hat{q}_{k j}\right)\right\} \\
\frac{\partial x}{m=1} \phi_{m} h_{m}+
\end{array}\right\}
\end{gathered}
$$

where $h_{m}, q_{x m}$, and $q_{y m}$ are values of potential and derivatives in directions $x$ and $y$ in supporting nodes in the neighbourhood of corresponding virtual points.

Coefficients $\alpha_{k}$ is defined by Eq. (14). The backward difference is used to approximate the time derivatives of potential on the right side of Eq. (4) and we get the resulting recurrent system of equations to solve potential and its derivatives in every node. Equations of heat transfer are solved using the same RBIE method and also the similar algorithms as the potential flow.

Equations of flow and heat transfer are then coupled by the equations of state which gives the fluid density and viscosity as functions of temperature. The coupling scheme was realized by the sequential-iterative approach using the modified Pickard algorithm:

- Step1: Solution of the transfer equations

- Step2: Update fluid flow properties $\rho, \mu, K_{x}, K_{y}$

- Step3: Solution of potential flow

- Step4: Compute velocities of flow

- Step5: Test the convergence of the process

This modified scheme converges much faster then the classical Pickard algorithm (see [10]). 


\section{Numerical example}

The RBIEM model has been verified with the Elder problem (see [5]) of free geothermal convection. The 2D domain is vertically oriented rectangular area filled with a homogeneous isotropic porous medium (see Fig. 3). It is a free convection problem where fluid flow is driven purely by fluid density differences. The elevated temperature of $20^{\circ} \mathrm{C}$ decreases water density and creates a potentially unstable situation where denser fluid overlies less dense fluid which leads to upwelling of warm water and to the formation of thermal fingering. In homogeneous isotropic media, the onset of free geothermal convection can be determined by the value of Rayleigh number $R a$ (see e.g. [5]). This number is the ratio between buoyancy forces driving free convection and conductive forces. The dimensionless thermal Rayleigh number can be defined as

$$
R a=\frac{K H c_{L} \Delta \rho_{L}}{K_{T}}
$$

where $K$ is the hydraulic conductivity, $H$ is the height of the model domain, $c_{L}$ is the specific heat of the liquid, $K_{T}$ is the thermal conductivity, and $\Delta \rho$ is the fluid density difference between the top and the bottom of the domain. The minimum value of the critical Rayleigh number $R a_{c r}=4 \pi^{2}$. It is valid if and only if the aspect ratio of the domain $a=L / H$ is an integer value (see [11]). According to theory, simulations with $R a<R a_{c r}$ are conductive, whereas systems with $R a>R a_{c r}$ exhibit convective and unstable flow.

The solved problem has the aspect ratio $a=L / H=2$ and therefore the critical Rayleigh number is $R a_{c r}=4 \pi^{2}=39.478$. The coefficients, used in the solution

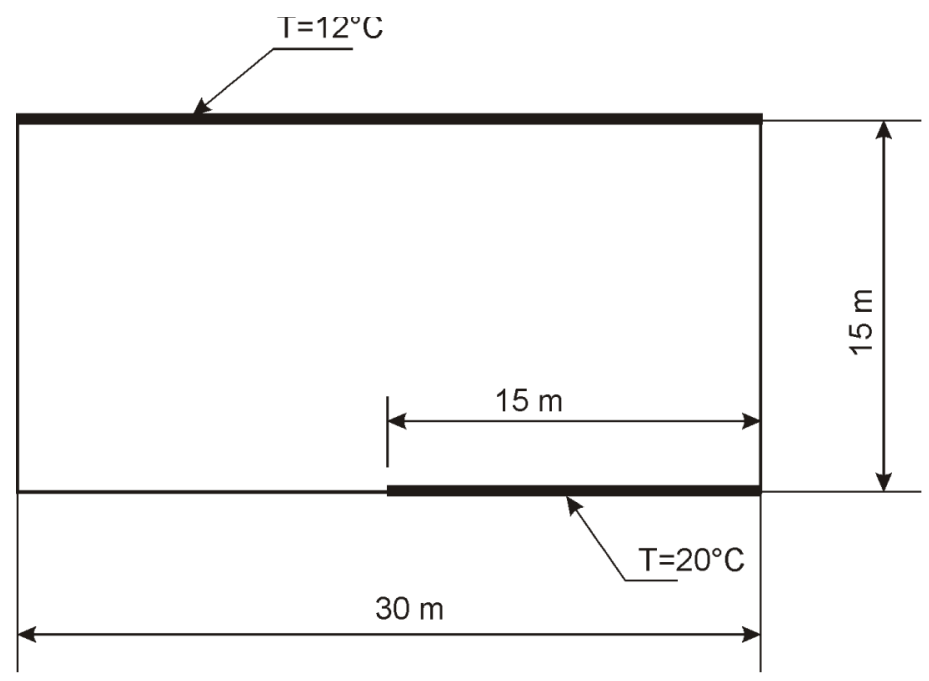

Figure 3: Elder problem. 
Table 1: Parameters of the solved problem.

\begin{tabular}{l|l|l}
\hline Quantity & Value & Unit \\
\hline Porosity & 0.1 & - \\
Hydraulic conductivity & $1.21 \mathrm{E}-04$ & $\mathrm{~ms}^{-1}$ \\
Bulk thermal conductivity & 1.49 & $\mathrm{Wm}^{-1} \mathrm{~K}^{-1}$ \\
Specific heat capacity & 4186 & $\mathrm{Jkg}^{-1} \mathrm{~K}^{-1}$ \\
Initial temperature & 12 & ${ }^{\circ} \mathrm{C}$ \\
\hline
\end{tabular}

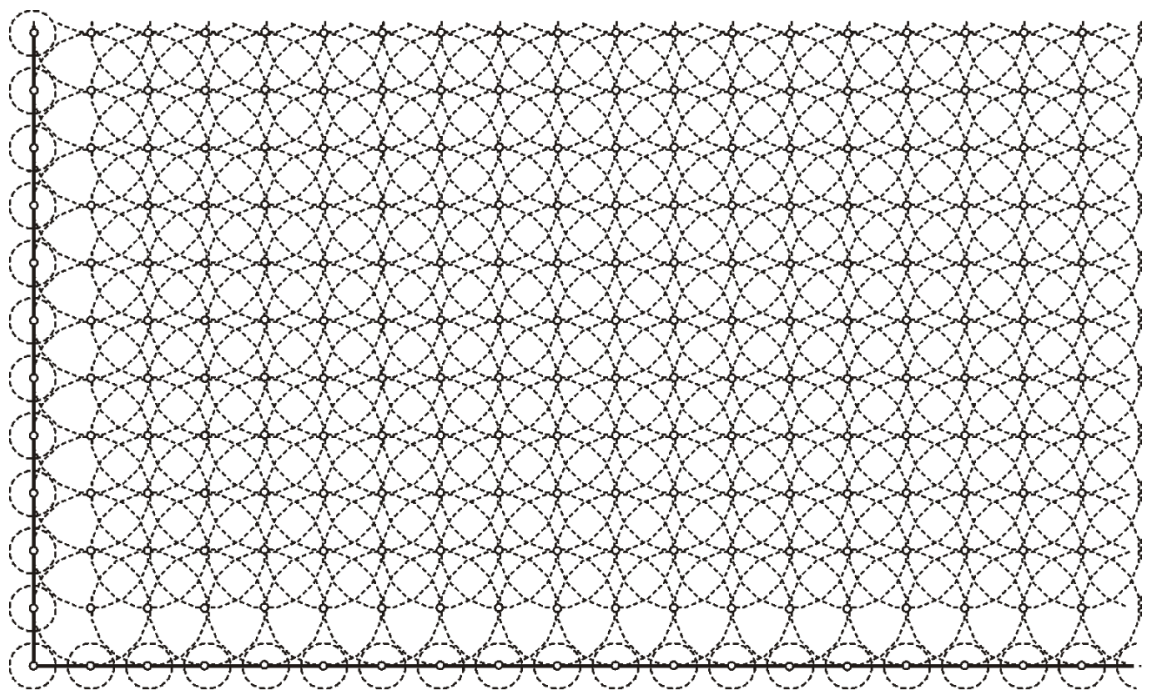

Figure 4: Detail of virtual network.

of the problem, are presented in Tab.1. The Rayleigh number computed for this problem is $R a=6.612<R a_{c r}$ and the problem is conductive and unstable flow should not occur. The domain is covered by 496 regularly distributed nodes and virtual networks were created around all node (see Fig. 4). The time step was 0.1 day. Fig. 5 shows the computed velocity field in 20 days and the increasing roll can be seen at the left part of the domain. The temperature distribution is presented in the form of isotherms at Fig. 6.

\section{Conclusions}

The possibility of RBIEM meshless method for modeling the density driven flow is presented in this paper. The research is at the beginning and the following study should be focused on the convective unstable situations models and on 


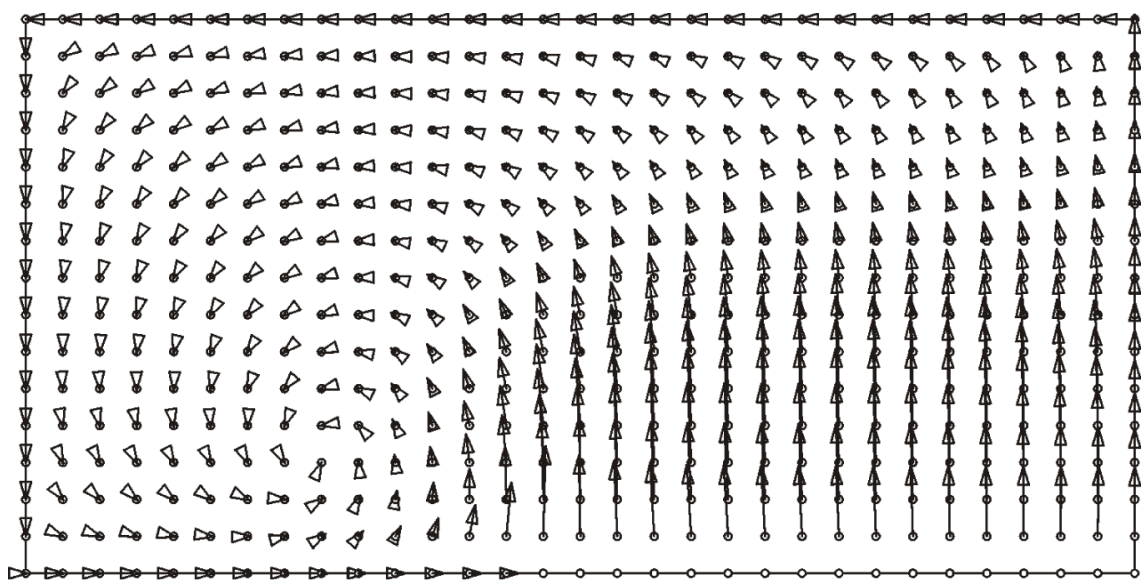

Figure 5: Resulting velocity field.

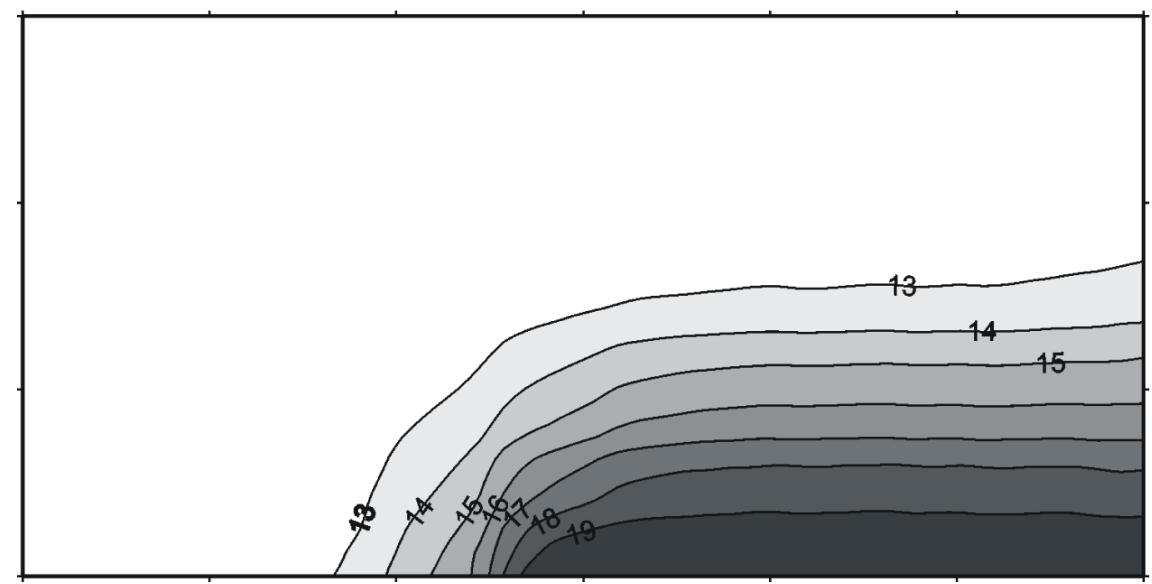

Figure 6: Isotherms.

the modification of the existing algorithms to enable distributed processing. It is necessary to choose suitable tools which give us ability to parallel solving of very large network systems which usually exist in practical solutions.

\section{Acknowledgements}

This contribution is the result of the project implementation: "Support of the Research and Development for Centre of Excellence in Transport Engineering" (ITMS: 26220120031) supported by the Research and Development Operational Programme funded by the ERDF. 


\section{References}

[1] K. Kovarik, Numerical models of groundwater pollution, Springer-Verlag, Berlin and New York, 2000.

[2] T. Zhu, J.D. Zhang, S.N. Atluri, A local boundary integral equation (LBIE) method in computational mechanics and a meshless discretization approach, Comput.Mech. 21, pp. 223-235, 1998.

[3] T.T. Bui, V. Popov, A meshless solution to two-dimensional convectiondiffusion problems, Engineering Analysis with Boundary Elements, 34, pp. 680-689, 2010.

[4] P. Ackerer, A. Younes, Efficient approximations for the simulation of density driven flow in porous media, Adv. Water Res., 31, pp. 15-27, 2008.

[5] T. Graf, Simulation of geothermal flow in deep sedimentary basins in Alberta, ERCB/AGS Open File Report 2009-11, 2009.

[6] P.W. Partridge, C. A. Brebbia, L.C. Wrobel, The Dual Reciprocity Boundary Element Method, CM Publications, Southampton, 1992.

[7] E.J. Kansa, Multiquadrics - A Scattered Data Approximation Scheme with Application to Computational Fluid Dynamics, Comput. Math. Appl., 19, pp. 127-145, 1990.

[8] C. Shu, H. Ding, K.S. Yeo, Local Radial Basis Function-based Differential Quadrature Method and its Application to Solve Two-dimensional Incompressible Navier-Stokes Equations, Comput. Meth. Appl. Mech. Eng., 192, pp. 941-954, 2003.

[9] M. Degham, A. Ghesmati, Numerical simulation of two-dimensional sineGordon solitons via a local weak meshless technique based on the radial point interpolation method (RPIM), Computer Physics Communication, 181, pp. 772-786, 2009.

[10] P. Ackerer, A. Younes, M. Mancip, A new coupling algorithm for densitydriven flow in porous media, Geophys. Res. Lett., 31, L12506, 2004.

[11] J.P. Caltagirone, Convection in a porous medium, Convective transport and instability phenomena, pp. 199-232, ed. J. Zierep, H. Oertel, Braunsche Hofbuchdruckerei und Verlag, Karlsruhe, 1982. Comput. Math. Appl., 19, pp. 127-145, 1990. 